\title{
POROSITY AND MODELS WITH DISCRETE INNOVATIONS
}

\author{
ALEXANDER J. ZASLAVSKI
}

Received 28 March 2004

In our previous work, we obtained sufficient conditions for the existence of trajectories with unbounded consumption for a model of economic dynamics with discrete innovations. In this paper, using the porosity notion, we show that for most models these conditions hold.

\section{Introduction}

We consider the model with discrete innovations introduced in [6] and studied in $[10,11$, $12,13]$. In this model, the state of the economy is determined by a set of operating technologies, a collection of funds corresponding to these technologies, and a set of known, but as yet not implemented technologies. To introduce a new technology, expenditures of the already utilized types of funds are required. As a result of these expenditures, the new technology at the next instant of time will be introduced into action with a certain initial reserve of new funds.

We consider a single-product economy which deals with two production factors: labor $L$ and funds $K$. The time is assumed to be discrete and the amount of labor is constant and equal to unity. The state of the economy is determined by a set of operating technologies, a collection of funds corresponding to these technologies, and a set of known, but as yet not implemented technologies.

A technology is a pair $(f, v)$ where $f$ is a production function of two variables $K, L$ and $v \in[0,1)$. Possessing at time $t$ funds $K$ and labor resources $L$, the economy utilizing the technology $(f, v)$ will produce during a unit time interval, a product in the amount of $f(K, L)$. Moreover, at time $t+1$ the economy will still have in its possession the used old funds in the amount of $v K$.

To introduce a new technology, expenditures of the already utilized types of funds are required. As a result of these expenditures, the new technology at the next instant of time will be introduced into action with a certain initial reserve of the new funds.

Let $I=\{0,1, \ldots\}$ and $\left\{\left(f^{i}, v^{i}\right): i \in I\right\}$ be the set of all technologies which can be utilized in the production process. At time $t \in I$ the state of the economy is given in the form

$$
\left(I_{0}^{t}, I_{n}^{t},\left(K_{t}^{i}, C_{t}^{i}\right)\left(i \in I_{0}^{t}\right)\right),
$$


where $I_{0}^{t}$ is a finite set of numbers (indices) of technologies introduced by the time $t, I_{n}^{t}$ is the set of numbers of technologies which are available in principle but not introduced, and $K_{t}^{i}, C_{t}^{i} \geq 0$ are the funds and consumption of the $i$ th type available at time $t$ which correspond to the technology with the number $i$. We will assume that at time $t$ the following information is available:

$$
\left(K^{i}\right),\left(s^{i j}\right) \quad\left(i \in I_{n}^{t}, j \in I_{n}^{t} \cup I_{0}^{t}\right),
$$

where $s^{i j} \geq 0$ is the expenditure of the $j$ th funds required for introduction of the $i$ th technology and $K^{i}>0$ is the initial amount of the $i$ th fund which is obtained at the initial time of utilization of the $i$ th technology. At time $t+1$ the economy may pass over to the state

$$
\left(I_{0}^{t+1}, I_{n}^{t+1},\left(K_{t+1}^{i}, C_{t+1}^{i}\right)\left(i \in I_{0}^{t+1}\right)\right)
$$

for which

$$
I_{0}^{t} \subset I_{0}^{t+1} \subset I_{0}^{t} \cup I_{n}^{t}, \quad I_{0}^{t+1} \backslash I_{0}^{t} \subset\left\{i \in I_{n}^{t}: s^{i j}=0 \forall j \in I_{n}^{t}\right\},
$$

and the numbers $L_{t}^{i} \geq 0, i \in I_{0}^{t}$ are determined such that

$$
\begin{gathered}
\sum_{i \in I_{0}^{t}} L_{t}^{i} \leq 1, \\
K_{t+1}^{j} \geq v^{j} K_{t}^{j} \quad \forall j \in I_{0}^{t}, \\
K_{t+1}^{i}=K^{i}, \quad C_{t+1}^{i}=0, \quad i \in I_{0}^{t+1} \backslash I_{0}^{t}, \\
K_{t+1}^{j}-v^{j} K_{t}^{j}+C_{t+1}^{j}+\sum_{i \in I_{0}^{t+1} \backslash I_{0}^{t}} s^{i j} \leq f^{j}\left(K_{t}^{j}, L_{t}^{j}\right) \quad \forall j \in I_{0}^{t} .
\end{gathered}
$$

(We assume here that the result of a summation over an empty set equals zero.) Note that in the model under consideration the newly produced product is used for consumption and expenditures related to an introduction of new technologies. Sometimes the state of the economy at time $t$ will be written in the form

$$
\left(I_{0}^{t}, I_{n}^{t},\left(K_{t}^{i}, C_{t}^{i}, L_{t}^{i}\right)\left(i \in I_{0}^{t}\right)\right)
$$

where $\left(L_{t}^{i}\right)\left(i \in I_{0}^{t}\right)$ is the distribution of labor resources at time $t$. We do it in the case when some information about this distribution is required. When describing a trajectory of the model, we would also include into its description the corresponding sequence of distributions of labor resources, most often only in the case when some information about these resources is required. However, in any case, a definite sequence of distributions of labor resources is always associated with a trajectory of the model.

Denote by $R_{+}^{l}$ the cone of elements of the Euclidean space $R^{l}$ with nonnegative coordinates. Below all the technologies under consideration $(f, v)$ will assume to be such that $f: R_{+}^{2} \rightarrow R_{+}$be a continuous, superlinear (superadditive, positively homogeneous) 
function,

$$
\begin{gathered}
f(0,1)=f(1,0)=0, \\
f(x, 1)<f(\lambda x, 1)<\lambda f(x, 1) \text { for each } \lambda>1 \text { and each } x>0,
\end{gathered}
$$

and there exists $X \in R_{+}$such that $f(1, X)>1-v$.

Let $(f, v)$ be a technology. It is easy to see that there exists a unique number $x(f, v)>0$ such that

$$
f(x(f, v), 1)=(1-v) x(f, v) .
$$

For $x_{0}>0$ the inequality $f\left(x_{0}, 1\right)>(1-v) x_{0}$ holds if and only if $x_{0}<x(f, v)$ and the sequence

$$
x_{t}=v x_{t-1}+f\left(x_{t-1}, 1\right), \quad t=1,2, \ldots
$$

converges to $x(f, v)$ as $t \rightarrow \infty$. Evidently $x(f, v)$ is a characteristic of the technology $(f, v)$ which evaluates its production capabilities.

The technology $(f, v)$ is associated with a dynamic model of the economy whose trajectory is a sequence $\left(K_{t}, C_{t}\right), t=0,1, \ldots$, where $K_{t}, C_{t} \geq 0$ are the funds and consumption available at time $t$ which satisfy

$$
K_{t+1}-v K_{t} \geq 0, \quad K_{t+1}-v K_{t}+C_{t+1} \leq f\left(K_{t}, 1\right)
$$

for all $t=0,1, \ldots$.

It is easy to see that for any model trajectory $\left(K_{t}, C_{t}\right), t=0,1, \ldots$, we have

$$
\limsup _{t \rightarrow \infty}\left(v K_{t}+f\left(K_{t}, 1\right)\right) \leq x(f, v), \quad \limsup _{t \rightarrow \infty} K_{t} \leq x(f, v) .
$$

Moreover, for any initial state of the model $\left(K_{0}, C_{0}\right)$ with $K_{0}>0$, there exists a trajectory $\left(K_{t}, C_{t}\right), t=0,1, \ldots$ such that $K_{t} \rightarrow x(f, v)$ as $t \rightarrow \infty$.

Let $X=\left(K_{t}, C_{t}\right)(t \in I)$ be a model trajectory. Set

$$
w(X)=\limsup _{T \rightarrow \infty} T^{-1} \sum_{t=0}^{T-1} C_{t} .
$$

Evidently $w(X) \leq x(f, v)$. Set

$$
w(f, v)=\sup \{w(X): X \text { is a model trajectory }\} .
$$

The number $w(f, v)$ is a characteristic of the technology $(f, v)$ which evaluates its consumption capabilities.

It is easy to verify that the following result is true.

Proposition 1.1. There exists a number $h(f, v) \in(0,1)$ such that

$$
\begin{gathered}
\lim _{x \rightarrow \infty}(h(f, v) f(1, x)) \in(1-v, \infty], \\
(1-h(f, v)) f(x(h(f, v) f, v), 1)=w(f, v)>0 .
\end{gathered}
$$




\section{Existence of trajectories with unbounded consumption}

Consider the model with discrete innovations introduced in Section 1. We assume that

$$
s^{i j}=0 \quad \forall i, j \in I \text { satisfying } j \geq i,
$$

and that for any state of the model $\left(I_{0}^{t}, I_{n}^{t},\left(K_{t}^{i}, C_{t}^{i}\right)\left(i \in I_{0}^{t}\right)\right)$ at any instant of time $t$ the relation

$$
\sup \left\{i: i \in I_{0}^{t}\right\}+1 \in I_{n}^{t}
$$

holds.

It is well known that for a model with a finite number of technologies all trajectories are bounded. For the model with discrete innovations a finite number of technologies can be introduced by time $t$, where $t=0,1, \ldots$, but the set of all technologies which can be utilized in the production process is infinite.

In [10], we studied the existence of model trajectories with unbounded consumption and established the following result.

Theorem 2.1. Let $\sup \left\{w\left(f^{i}, v^{i}\right): i \in I\right\}=\infty$ and

$$
\sup _{p \in I}\left\{\sum_{i \in I} s^{p i}\left(x\left(f^{i}, v^{i}\right)\left(1-v^{i}\right)\right)^{-1}\right\}<1 .
$$

Assume that $\left(I_{0}^{0}, I_{n}^{0},\left(K_{0}^{i}, C_{0}^{i}\right)\left(I \in I_{0}^{0}\right)\right)$ is an initial state of the economy such that $K_{0}^{i}>0$, $i \in I_{0}^{0}$, and

$$
s^{p j}=0 \quad \text { for any } p>p(0):=\sup \left\{i: i \in I_{0}^{0}\right\} \text { and any } j \in\{0, \ldots, p(0)\} \backslash I_{0}^{0} .
$$

Then there exists a model trajectory

$$
\left(I_{0}^{t}, I_{n}^{t},\left(K_{t}^{i}, C_{i}^{t}\right)\left(I \in I_{0}^{t}\right)\right) \quad(t \in I)
$$

such that

$$
0<\sup \left\{C_{t}^{i}: i \in I_{0}^{t}\right\} \rightarrow \infty \text { as } t \rightarrow \infty .
$$

Note that inequality (2.3) means that the expenditures of the $i$ th funds required for introduction of the technology $\left(f^{p}, v^{p}\right)$ should be small compared to the value

$$
x\left(f^{i}, v^{i}\right)\left(1-v^{i}\right)=f^{i}\left(x\left(f^{i}, v^{i}\right), 1\right)
$$

which characterizes the production capabilities of the technology $\left(f^{i}, v^{i}\right)$.

We consider a particular case of the model such that for each $i, j \in I$ the inequality $s^{i j}>0$ holds if and only if $i=j+1$. This assumption means that for introduction of the $(i+1)$ th technology we only need expenditures of the $i$ th fund.

In this case inequality (2.3) is equivalent to the following inequality:

$$
\sup _{i \in I}\left\{s^{(i+1) i}\left(x\left(f^{i}, v^{i}\right)\left(1-v^{i}\right)\right)^{-1}\right\}<1 .
$$


Let $i \in I$ and the $i$ th technology be introduced at time $t_{0}$. Assume that $K^{i}<x\left(f^{i}, v^{i}\right)$. This inequality means that the initial amount of the $i$ th fund is less than the characteristic of the technology $\left(f^{i}, v^{i}\right)$ which evaluates its production capabilities. It is easy to see that for each instant of time $t>t_{0}, K_{t}^{i}<x\left(f^{i}, v^{i}\right)$, and $f^{i}\left(K_{t}^{i}, 1\right)<f^{i}\left(x\left(f^{i}, v^{i}\right), 1\right)$. Therefore the inequality

$$
s^{(i+1) i}<f^{i}\left(x\left(f^{i}, v^{i}\right), 1\right)
$$

is necessary for implementation of the $(i+1)$ th technology.

If $I_{0}^{0}=\{0\}$ and inequality (2.9) is not true for some integer $i \geq 0$, then only a finite number of technologies can be implemented. Therefore it is natural to consider a class of models such that inequality (2.9) holds for all integers $i \geq 0$. By Theorem 2.1, in order to construct trajectories with unbounded consumption, we need inequality (2.8) which is stronger than (2.9). In this paper we consider two classes of models which satisfy inequality (2.9) and show that for most of the models in these spaces inequality (2.8) is valid.

When we say that most of the elements of a complete metric space $Y$ enjoy a certain property, we mean that the set of points which have this property contains a $G_{\delta}$ everywhere dense subset of $Y$. In other words, this property holds generically. Such an approach, when a certain property is investigated for the whole space $Y$ and not just for a single point in $Y$, has already been successfully applied in many areas of analysis. See, for example, $[2,3,4,5,7]$ and the references mentioned therein.

We use the concept of porosity which will enable us to obtain even more refined results.

In this paper, we consider two metric spaces of models which satisfy inequality (2.9). Using the porosity notion we show that for most models in these spaces inequality (2.8) is true. Combined with Theorem 2.1 our results imply that for most of models in these spaces there exist trajectories with unbounded consumption.

We now recall the notion of porosity $[1,4,7,8,9]$.

Let $(Y, d)$ be a metric space. We denote by $B(y, r)$ the closed ball of center $y \in Y$ and radius $r>0$. A subset $E \subset Y$ is called porous in $(Y, d)$ if there exist $\alpha \in(0,1)$ and $r_{0}>0$ such that for each $r \in\left(0, r_{0}\right]$ and each $y \in Y$ there exists $z \in Y$ for which

$$
B(z, \alpha r) \subset B(y, r) \backslash E .
$$

Other notions of porosity have been used in the literature $[1,8,9]$. We use the rather strong notion which appears in $[4,7]$.

\section{The first main result}

We use the convention that $\infty / \infty=1$. Let $\left\{\left(f^{i}, v^{i}\right): i \in I\right\}$ be a set of technologies which satisfy the assumptions introduced in introduction.

Denote by $\mathscr{A}$ the set of all sequences $\left\{\sigma_{i}\right\}_{i=0}^{\infty}$ of positive numbers such that

$$
\sigma_{i} \leq f^{i}\left(x\left(f^{i}, v^{i}\right), 1\right) \quad \forall i \in I .
$$


Let $\left\{\sigma_{i}\right\}_{i=0}^{\infty} \in \mathscr{A}$. This sequence generates a model with the set of technologies $\left\{\left(f^{i}, v^{i}\right)\right.$ : $i \in I\}$ such that for each $i, j \in I$

$$
s^{j i}=0 \quad \text { if } j \neq i+1, \quad s^{(i+1) i}=\sigma_{i} .
$$

We equip the set $\mathscr{A}$ with a metric. For each $\left\{\alpha_{i}\right\}_{i=0}^{\infty},\left\{\beta_{i}\right\}_{i=0}^{\infty} \in \mathscr{A}$ set

$$
\begin{aligned}
& \tilde{d}\left(\left\{\alpha_{i}\right\}_{i=0}^{\infty},\left\{\beta_{i}\right\}_{i=0}^{\infty}\right)=\sup \left\{\left|\ln \left(\frac{\alpha_{i}}{\beta_{i}}\right)\right|: i \in I\right\}, \\
& d\left(\left\{\alpha_{i}\right\}_{i=1}^{\infty},\left\{\beta_{i}\right\}_{i=0}^{\infty}\right)=\tilde{d}\left(\left\{\alpha_{i}\right\}_{i=0}^{\infty},\left\{\beta_{i}\right\}_{i=0}^{\infty}\right)\left(1+\tilde{d}\left(\left\{\alpha_{i}\right\}_{i=0}^{\infty},\left\{\beta_{i}\right\}_{i=0}^{\infty}\right)\right)^{-1} .
\end{aligned}
$$

It is not difficult to see that $d: \mathscr{A} \times \mathscr{A} \rightarrow R^{1}$ is a metric and that the metric space $(\mathscr{A}, d)$ is complete.

Denote by $\mathscr{F}_{A}$ the set of all $\left\{\sigma_{i}\right\}_{i=0}^{\infty} \in \mathscr{A}$ such that

$$
\sup \left\{\sigma_{i} f^{i}\left(x\left(f^{i}, v^{i}\right), 1\right)^{-1}: i \in I\right\}<1 .
$$

Theorem 3.1. $\mathscr{A} \backslash \mathscr{F}_{A}$ is a porous subset of $(\mathscr{A}, d)$.

Proof. Set

$$
\alpha=\frac{1}{16} \text {. }
$$

Let $\left\{\sigma_{i}\right\}_{i=0}^{\infty} \in \mathscr{A}$ and $r \in(0,1]$. Set

$$
\lambda=\exp (2 \alpha r)
$$

By (3.7) and (3.6)

$$
1<\lambda<e^{r / 4}
$$

Define

$$
\bar{\sigma}_{i}=\sigma_{i} \lambda^{-1}, \quad i \in I .
$$

Clearly $\left\{\bar{\sigma}_{i}\right\}_{i=0}^{\infty} \in \mathscr{A}$.

Assume that

$$
\left\{\Delta_{i}\right\}_{i=0}^{\infty} \in \mathscr{A}, \quad d\left(\left\{\Delta_{i}\right\}_{i=0}^{\infty},\left\{\bar{\sigma}_{i}\right\}_{i=0}^{\infty}\right) \leq \alpha r .
$$

It follows from (3.9), (3.3), and (3.8) that

$$
d\left(\left\{\sigma_{i}\right\}_{i=0}^{\infty},\left\{\bar{\sigma}_{i}\right\}_{i=0}^{\infty}\right) \leq \ln \lambda \leq \frac{r}{4} .
$$

Together with (3.10) and (3.6), this implies that

$$
d\left(\left\{\Delta_{i}\right\}_{i=0}^{\infty},\left\{\sigma_{i}\right\}_{i=0}^{\infty}\right) \leq d\left(\left\{\Delta_{i}\right\}_{i=0}^{\infty},\left\{\bar{\sigma}_{i}\right\}_{i=0}^{\infty}\right)+d\left(\left\{\sigma_{i}\right\}_{i=0}^{\infty},\left\{\bar{\sigma}_{i}\right\}_{i=0}^{\infty}\right) \leq \alpha r+\frac{r}{4}<r .
$$


By (3.10), (3.3), and (3.6)

$$
\begin{aligned}
\tilde{d}\left(\left\{\Delta_{i}\right\}_{i=0}^{\infty},\left\{\bar{\sigma}_{i}\right\}_{i=0}^{\infty}\right) & =d\left(\left\{\Delta_{i}\right\}_{i=0}^{\infty},\left\{\bar{\sigma}_{i}\right\}_{i=0}^{\infty}\right)\left(1-d\left(\left\{\Delta_{i}\right\}_{i=0}^{\infty},\left\{\bar{\sigma}_{i}\right\}_{i=0}^{\infty}\right)\right)^{-1} \\
& \leq \alpha r(1-\alpha r)^{-1} \leq\left(\frac{5}{4}\right) \alpha r .
\end{aligned}
$$

Let $i \in I$. It follows from (3.3), (3.13), (3.9), (3.7), and (3.1) that

$$
\begin{aligned}
\Delta_{i} & \leq \bar{\sigma}_{i} \exp \left(\left(\frac{5}{4}\right) \alpha r\right)=\lambda^{-1} \exp \left(\left(\frac{5}{4}\right) \alpha r\right) \sigma_{i} \\
& \leq \exp \left(-\frac{\alpha r}{2}\right) \sigma_{i} \leq \exp \left(-\frac{\alpha r}{2}\right) f^{i}\left(x\left(f^{i}, v^{i}\right), 1\right) .
\end{aligned}
$$

Hence

$$
\sup \left\{\Delta_{i}\left(f^{i}\left(x\left(f^{i}, v^{i}\right), 1\right)\right)^{-1}: i \in I\right\} \leq \exp \left(-\frac{\alpha r}{2}\right)
$$

and $\left\{\Delta_{i}\right\}_{i=0}^{\infty} \in \mathscr{F}_{A}$. Theorem 3.1 is proved.

\section{The second main result}

Let $f, g: R_{+}^{2} \rightarrow R^{1}$. We say that $f \leq g$ if $f(x) \leq g(x)$ for each $x \in R_{+}^{2}$.

Denote by $\mathscr{B}$ the set of all collections

$$
b=\left(\left\{\left(f^{i}, v^{i}\right): i \in I\right\},\left\{\sigma_{i}\right\}_{i=0}^{\infty}\right)
$$

such that $\left(f^{i}, v^{i}\right), i \in I$ are technologies which satisfy assumptions introduced in Section 1 and

$$
\sigma_{i} \in\left(0, f^{i}\left(x\left(f^{i}, v^{i}\right), 1\right)\right], \quad i \in I
$$

Any

$$
b=\left(\left\{\left(f^{i}, v^{i}\right): i \in I\right\},\left\{\sigma_{i}\right\}_{i=0}^{\infty}\right)
$$

from $\mathscr{B}$ generates a model denoted by $\mathcal{M}(b)$ with the set of technologies $\left\{\left(f^{i}, v^{i}\right): i \in I\right\}$ such that for each $i, j \in I$,

$$
s^{j i}=0 \quad \text { if } j \neq i+1, \quad s^{(i+1) i}=\sigma_{i} .
$$

We assume that an infimum of an empty set is zero.

We equip the set $\mathscr{B}$ with a metric.

For each

$$
a_{1}=\left(\left\{\left(f_{1}^{i}, v_{1}^{i}\right): i \in I\right\},\left\{\sigma_{i 1}\right\}_{i=0}^{\infty}\right), \quad a_{2}=\left(\left\{\left(f_{2}^{i}, v_{2}^{i}\right): i \in I\right\},\left\{\sigma_{i 2}\right\}_{i=0}^{\infty}\right) \in \mathscr{B}
$$


set

$$
\begin{gathered}
\tilde{d}\left(a_{1}, a_{2}\right)=\inf \left\{\gamma \geq 0: e^{-\gamma}\left(1-v_{1}^{i}\right) \leq\left(1-v_{2}^{i}\right) \leq e^{\gamma}\left(1-v_{1}^{i}\right), i \in I,\right. \\
\left.e^{-\gamma} \sigma_{i 1} \leq \sigma_{i 2} \leq e^{\gamma} \sigma_{i 1}, i \in I, e^{-\gamma} f_{1}^{i} \leq f_{2}^{i} \leq e^{\gamma} f_{2}^{i}, i \in I\right\}, \\
d\left(a_{1}, a_{2}\right)=\tilde{d}\left(a_{1}, a_{2}\right)\left(1+\tilde{d}\left(a_{1}, a_{2}\right)\right)^{-1} .
\end{gathered}
$$

Clearly $(\mathscr{B}, d)$ is a metric space.

Denote by $\mathscr{F}_{\mathscr{B}}$ the set of all $b=\left(\left\{\left(f^{i}, v^{i}\right): i \in I\right\},\left\{\sigma_{i}\right\}_{i=0}^{\infty}\right) \in \mathscr{B}$ such that

$$
\sup _{i \in I}\left\{\sigma_{i}\left(f^{i}\left(x\left(f^{i}, v^{i}\right), 1\right)\right)^{-1}\right\}<1 .
$$

Theorem 4.1. $\mathscr{B} \backslash \mathscr{F}_{\mathscr{B}}$ is a porous subset of $(\mathscr{B}, d)$.

Proof. Set

$$
\alpha=32^{-1}
$$

Let

$$
b=\left(\left\{\left(f^{i}, v^{i}\right): i \in I\right\},\left\{\sigma_{i}\right\}_{i=0}^{\infty}\right) \in \mathscr{B}, \quad r \in(0,1] .
$$

Set

$$
\lambda=e^{8 \alpha r}
$$

Define

$$
\bar{b}=\left(\left\{\left(\bar{f}^{i}, \bar{v}^{i}\right): i \in I\right\},\left\{\bar{\sigma}_{i}\right\}_{i=0}^{\infty}\right) \in \mathscr{B}
$$

as follows:

$$
\begin{gathered}
\bar{\sigma}_{i}=\sigma_{i} \lambda^{-1}, \quad i \in I, \quad \bar{v}^{i}=v^{i}, \quad i \in I, \\
\bar{f}^{i}=\lambda f^{i}, \quad i \in I .
\end{gathered}
$$

In view of (4.13), (4.14), (4.6), (4.11), and (4.9),

$$
d(b, \bar{b}) \leq \ln \lambda \leq \frac{r}{4} .
$$

Assume that

$$
\begin{gathered}
d=\left(\left\{\left(g^{i}, u^{i}\right): i \in I\right\},\left\{\Delta_{i}\right\}_{i=0}^{\infty}\right) \in \mathscr{B}, \\
d(a, \bar{b}) \leq \alpha r .
\end{gathered}
$$

Equations (4.15), (4.16), and (4.9) imply that

$$
d(a, b) \leq d(a, \bar{b})+d(\bar{b}, b) \leq \alpha r+\frac{r}{4}<r .
$$


Let $i \in I$. By (4.6), (4.16), and (4.9),

$$
\tilde{d}(a, \bar{b})=d(a, \bar{b})(1-d(a, \bar{b}))^{-1} \leq \alpha r(1-\alpha r)^{-1} \leq\left(\frac{5}{4}\right) \alpha r .
$$

In view of (4.16), (4.18), (4.6), (4.12), (4.14), (4.13), and (4.11),

$$
\begin{aligned}
& \Delta_{i} \leq \bar{\sigma}_{i} \exp \left(\left(\frac{5}{4}\right) \alpha r\right) \leq \lambda^{-1} \sigma_{i} \exp \left(\left(\frac{5}{4}\right) \alpha r\right) \leq \sigma_{i} \exp (-6 \alpha r), \\
& \left(1-v^{i}\right) \exp \left(\left(\frac{5}{4}\right) \alpha r\right) \geq\left(1-u^{i}\right) \geq\left(1-v^{i}\right) \exp \left(-\left(\frac{5}{4}\right) \alpha r\right), \\
& g^{i} \geq \exp \left(-\left(\frac{5}{4}\right) \alpha r\right) \bar{f}^{i} \geq \exp \left(-\left(\frac{5}{4}\right) \alpha r\right) \lambda f^{i} \geq \exp (6 \alpha r) f^{i} .
\end{aligned}
$$

We have

$$
f^{i}\left(x\left(f^{i}, v^{i}\right), 1\right)=\left(1-v^{i}\right) x\left(f^{i}, v^{i}\right) .
$$

It follows from (4.19) and (4.20) that

$$
\begin{aligned}
g^{i}\left(x\left(f^{i}, v^{i}\right), 1\right) & \geq \exp (6 \alpha r) f^{i}\left(x\left(f^{i}, v^{i}\right), 1\right) \\
& =\exp (6 \alpha r)\left(1-v^{i}\right) x\left(f^{i}, v^{i}\right) \\
& \geq\left(1-u^{i}\right) \exp \left(-\left(\frac{5}{4}\right) \alpha r\right) \exp (6 \alpha r) x\left(f^{i}, v^{i}\right) .
\end{aligned}
$$

This inequality implies that

$$
x\left(g^{i}, u^{i}\right)>x\left(f^{i}, v^{i}\right) .
$$

It follows from (4.19), (4.2), and (4.22) that

$$
\begin{aligned}
\Delta_{i} & \leq \sigma_{i} \exp (-6 \alpha r) \leq \exp (-6 \alpha r) f^{i}\left(x\left(f^{i}, v^{i}\right), 1\right) \\
& \leq \exp (-6 \alpha r) f^{i}\left(x\left(g^{i}, u^{i}\right), 1\right) \leq \exp (-6 \alpha r) g^{i}\left(x\left(g^{i}, u^{i}\right), 1\right) .
\end{aligned}
$$

Thus

$$
\sup \left\{\Delta_{i}\left(g^{i}\left(x\left(g^{i}, u^{i}\right), 1\right)\right)^{-1}: i \in I\right\} \leq \exp (-6 \alpha)
$$

and $d \in \mathscr{F}_{\mathscr{B}}$. Theorem 4.1 is proved.

\section{References}

[1] Y. Benyamini and J. Lindenstrauss, Geometric Nonlinear Functional Analysis. Vol. 1, American Mathematical Society Colloquium Publications, vol. 48, American Mathematical Society, Rhode Island, 2000.

[2] S. Cobzaş, Generic existence of solutions for some perturbed optimization problems, J. Math. Anal. Appl. 243 (2000), no. 2, 344-356.

[3] F. S. De Blasi and J. Myjak, Generic flows generated by continuous vector fields in Banach spaces, Adv. Math. 50 (1983), no. 3, 266-280. 
[4] Sur la porosité de l'ensemble des contractions sans point fixe [On the porosity of the set of contractions without fixed points], ClR. Acad. Sci. Paris Sér. I Math. 308 (1989), no. 2, 51-54 (French).

[5] A. D. Ioffe and A. J. Zaslavski, Variational principles and well-posedness in optimization and calculus of variations, SIAM J. Control Optim. 38 (2000), no. 2, 566-581.

[6] V. L. Makarov, On dynamic models of economy and development of ideas of L. V. Kantorovich, Economics and Mathematical Methods 23 (1987), 10-24.

[7] S. Reich and A. J. Zaslavski, The set of divergent descent methods in a Banach space is $\sigma$-porous, SIAM J. Optim. 11 (2001), no. 4, 1003-1018.

[8] L. Zajíček, Porosity and $\sigma$-porosity, Real Anal. Exchange 13 (1987/1988), no. 2, 314-350.

[9] - On $\sigma$-porous sets in abstract spaces, to appear in Abstr. Appl. Anal.

[10] A. J. Zaslavski, On a model of economic dynamics with discrete innovations, Economics and Mathematical Methods 25 (1989), 679-684.

[11] Discrete innovations in dynamic models of economy, Siberian Math. J. 31 (1990), no. 4, 566-577.

[12] Optimization of the growth rates in models with discrete innovations, Siberian Math. J. 32 (1991), no. 5, 760-766.

[13] Asymptotic behavior of paths of a model with discrete innovations, Siberian Math. J. 33 (1992), no. 4, 608-617.

Alexander J. Zaslavski: Department of Mathematics, Technion - Israel Institute of Technology, 32000 Haifa, Israel

E-mail address: ajzasl@tx.technion.ac.il 


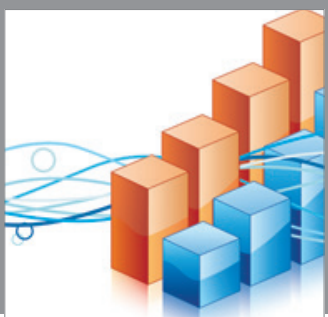

Advances in

Operations Research

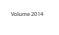

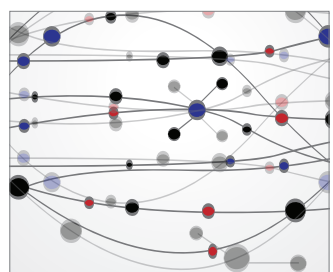

\section{The Scientific} World Journal
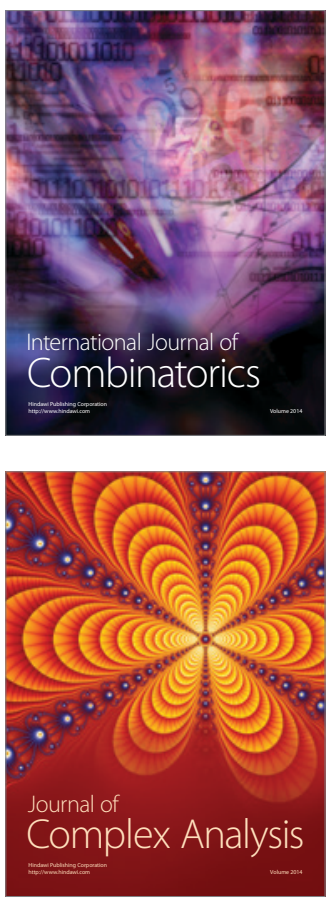

International Journal of

Mathematics and

Mathematical

Sciences
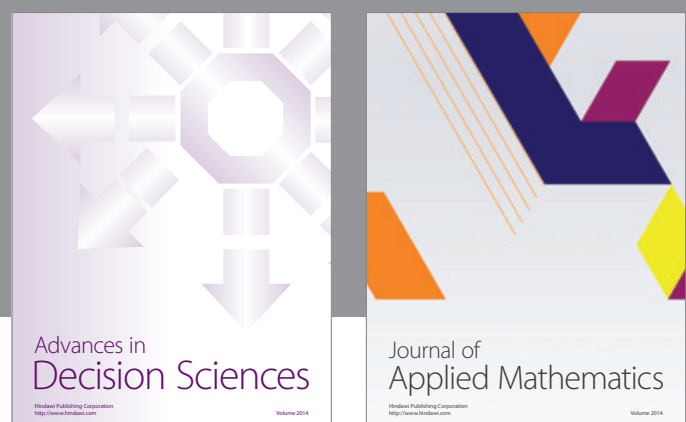

Journal of

Applied Mathematics
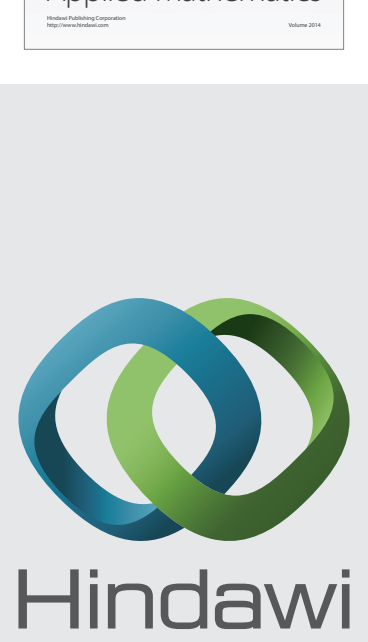

Submit your manuscripts at http://www.hindawi.com
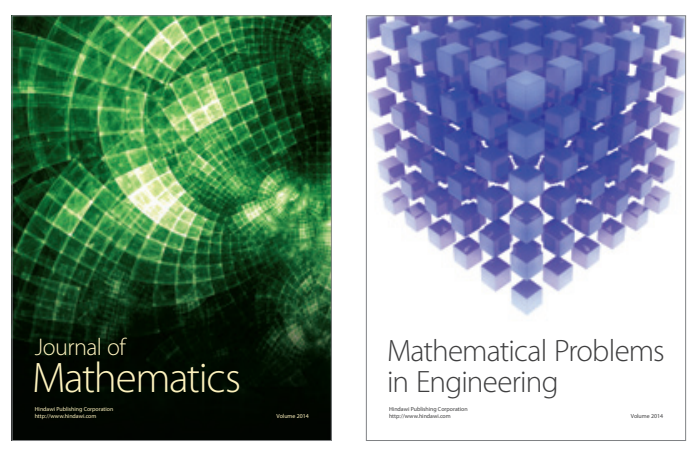

Mathematical Problems in Engineering
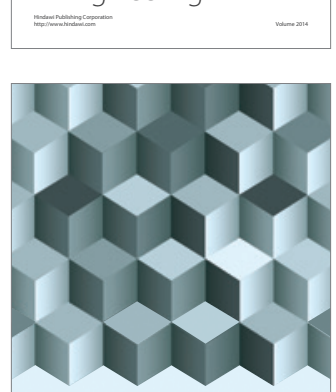

Journal of

Function Spaces
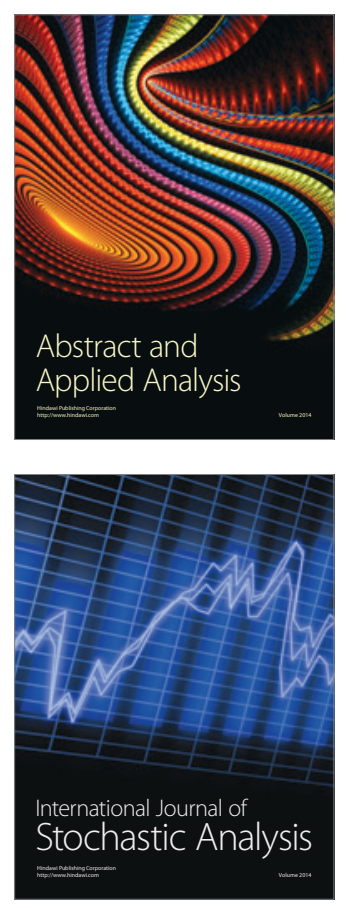

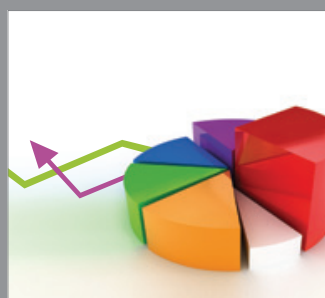

ournal of

Probability and Statistics

Promensencen
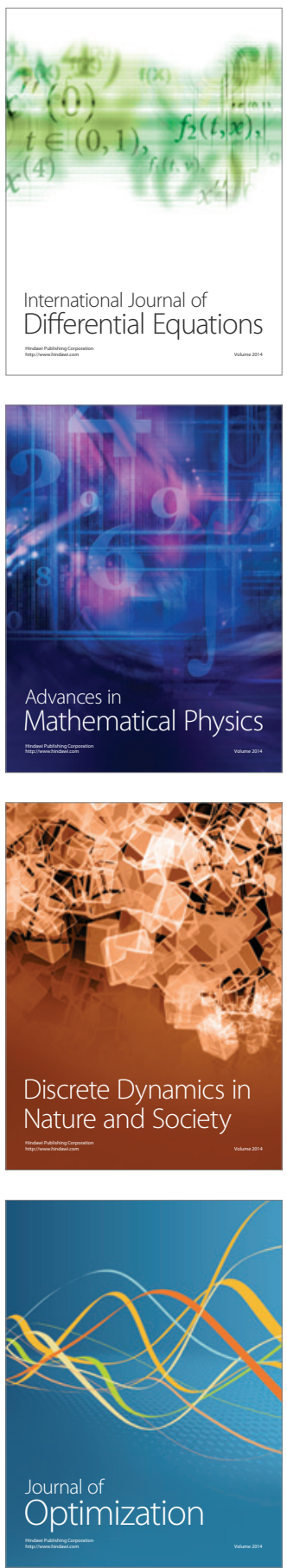